\title{
Totally anomalous systemic venous connection: where is the coronary sinus?
}

Received: 15 September 2017; Accepted: 1 October 2017; First published online: 10 January 2018

We read with interest the report by Miltner et al. We congratulate the authors for prenatal identification of an extremely rare anomaly of venous connections. ${ }^{1}$ It is unfortunate that the child did not survive, despite surgical atrial septectomy and construction of a modified Blalock-Taussig shunt to improve the flow of blood to the lungs. It is also unfortunate that the parents refused an autopsy to provide confirmation of the described findings. The authors elegantly utilised the discrepancy in size of the right and left ventricles so as to permit prenatal recognition of the suspected totally anomalous systemic venous connections. In our own review of this rare anomaly, we have emphasised the importance of recognising hypoplasia of right-sided cardiac chambers, particularly when associated with otherwise unexplained dilation of the left ventricle. ${ }^{2}$ In the case described by Miltner et al, it was the persistence of flow through the arterial duct that permitted flow of blood to the lungs and growth of the pulmonary arteries.

Their description, however, is surely lacking in information regarding the connection of the coronary sinus? As we sought to highlight in our review, it is essential that all the systemic veins, including the coronary sinus, must connect anomalously in order to justify the label of totally anomalous systemic venous connection. The authors also discussed the possibility of so-called heterotaxy syndrome, basing this solely on the external appearance of the right-sided atrial appendage. This is in keeping with the current practice of defining atrial arrangement. In our review, we also highlighted the problems in identifying isomerism of the atrial appendages, which is the essence of the cardiac features of heterotaxy syndrome. We have doubts, however, regarding the accuracy of such an interpretation when the right-sided atrium itself is

Correspondence to: Dr S. K. Gupta, Department of Cardiology, Cardiothoracic Centre, All India Institute of Medical Sciences, Room No. 9, 8th floor, New Delhi 110029, India. Tel: +91 112659 4944; E-mail: drsaurabhmd@gmail.com hypoplastic, as is the case in the setting of totally anomalous systemic venous connection, and particularly when there are no other features to suggest the presence of isomerism. This is more important, as we emphasised the problems of using the diagnostic label of anomalous systemic venous connection in hearts with isomeric atrial appendages. If both the atriums are morphologically left, then the systemic veins will self-evidently connect anomalously, irrespective of whether or not they are connected to the left-sided atrium. We agree, nonetheless, that the haemodynamic consequences are the same as in hearts with lateralised atrial arrangement.

\section{Acknowledgement}

None.

\section{Financial Support}

This research received no specific grant from any funding agency, commercial, or not-for-profit sectors.

Saurabb Kumar Gupta Department of Cardiology, All India Institute of Medical Sciences, New Delbi, India

Robert H. Anderson Institute of Genetic Medicine, Newcastle University Newcastle Upon Tyne, United Kingdom

\section{References}

1. Miltner B, Poncelet AJ, Barrea C. Prenatal diagnosis of isolated total anomalous systemic venous connection to the left atrium. Cardiol Young 2017; 27: 1394-1397.

2. Gupta SK, Juneja R, Anderson RH, Gulati GS, Devagourou V. Clarifying the anatomy and physiology of totally anomalous systemic venous connection. Ann Pediatr Cardiol 2017; 10: 269-277. 